\title{
TWEAK/Fn14 signaling axis mediates skeletal muscle atrophy and metabolic dysfunction
}

\section{Shuichi Sato, Yuji Ogura and Ashok Kumar*}

Department of Anatomical Sciences and Neurobiology, University of Louisville School of Medicine, Louisville, KY, USA

\section{Edited by:}

Timothy S. Zheng, Biogen Idec Inc., USA

\section{Reviewed by:}

Fulvio D'Acquisto, Queen Mary

University of London, UK

Takayuki Yoshimoto, Tokyo Medical

University, Japan

\section{*Correspondence:}

Ashok Kumar, Department of

Anatomical Sciences and

Neurobiology, University of Louisville

School of Medicine, 500 South

Preston Street, Louisville, KY 40202,

USA

e-mail: ashok.kumar@louisville.edu
Tumor necrosis factor-like weak inducer of apoptosis (TWEAK) through binding to its receptor fibroblast growth factor inducible 14 (Fn14) has been shown to regulate many cellular responses including proliferation, differentiation, apoptosis, inflammation, and fibrosis, under both physiological and pathological conditions. Emerging evidence suggests that TWEAK is also a major muscle wasting cytokine. TWEAK activates nuclear factor-kB signaling and proteolytic pathways such as ubiquitin-proteasome system, autophagy, and caspases to induce muscle proteolysis in cultured myotubes. Fn14 is dormant or expressed in minimal amounts in normal healthy muscle. However, specific atrophic conditions, such as denervation, immobilization, and starvation stimulate the expression of Fn14 leading to activation of TWEAK/Fn14 signaling and eventually skeletal muscle atrophy. TWEAK also causes slow- to fast-type fiber transition in skeletal muscle. Furthermore, recent studies suggest that TWEAK diminishes mitochondrial content and represses skeletal muscle oxidative phosphorylation capacity. TWEAK mediates these effects through affecting the expression of a number of genes and microRNAs. In this review article, we have discussed the recent advancements toward understanding the role and mechanisms of action of TWEAK/Fn14 signaling in skeletal muscle with particular reference to different models of atrophy and oxidative metabolism.

Keywords:TWEAK, Fn14, skeletal muscle, atrophy, oxidative phosphorylation

\section{INTRODUCTION}

Skeletal muscle is the largest tissue of the human body, which provides posture, ensures basic functions such as locomotion and respiration, and plays a vital role in whole body metabolism. Skeletal muscle is also one of the most highly plastic tissues in the body. A number of extracellular and intracellular signals have now been identified, which cause physiological adaptations including enhanced substrate metabolism, mitochondrial biogenesis, angiogenesis, muscle growth, and regeneration $(1,2)$. For example, exercise training causes improvement in muscle mass and contractility, and metabolic function resulting in enhanced force generation capacity and resistance to fatigability. Conversely, inactivity and many chronic disease states result in loss of skeletal muscle mass and metabolic dysfunction $(3,4)$. Maintenance of skeletal muscle mass and function are prerequisites for whole body health throughout life.

Skeletal muscle mass is maintained through a delicate balance between protein synthesis and degradation. Resistance exercise, hormones, and nutritional uptake increase rate of protein synthesis resulting in increased muscle mass (5). Insulin-like growth factor-1 (IGF-1)/phosphatidylinositol 3kinase (PI3K)/Akt/mTOR is one of the most important signaling pathways, which increases protein synthesis leading to skeletal muscle hypertrophy $(6,7)$. This pathway also inhibits muscle protein degradation through distinct mechanisms $(8,9)$. By contrast, many catabolic stimuli increase the activity of various signaling intermediates, such as extracellular-regulated kinase $1 / 2$
(ERK1/2), c-Jun-N-terminal kinase (JNK), p38 mitogen-activated protein kinase (MAPK), AMP-activated protein kinase (AMPK), and TNF receptor-associated factors (TRAFs) and transcription factors, such as nuclear factor- $\mathrm{kB}$ (NF- $\mathrm{KB})$, activator protein 1 (AP1), p53, FoxO1, and FoxO3a resulting in the activation of ubiquitin-proteasome system (UPS) and autophagy-lysosomal system (ALS), the two major proteolytic mechanisms in skeletal muscle $(1,10,11)$. Recent studies also suggest that activating transcription factor 4 (ATF4), growth arrest and DNA damageinducible $45 \alpha$ (GADD45 $\alpha$ ), histone deacetylase 4 (HDAC4), and myogenin mediate skeletal muscle atrophy under specific conditions (12-14). Furthermore, changes in the mitochondrial content, integrity, and function play a critical role in regulation of skeletal muscle mass and function $(15,16)$. The biogenesis of new mitochondria and clearance of defunct mitochondria are essential to meet cellular energy demand especially during endurance exercise and to protect from many chronic diseases such as diabetes, heart failure, obesity, and cancer $(17,18)$. Impairment in mitochondrial function is also an important facet of aged skeletal muscle. However, it is not yet clear whether dysfunctional mitochondria are a cause or consequence of aged skeletal muscle (19).

Classical proinflammatory cytokines, such as tumor necrosis factor- $\alpha(\mathrm{TNF} \alpha)$, interleukin-1 $\beta$ (IL-1 $\beta)$, IL-6, and interferon- $\gamma$ have been suggested as important mediators of catabolic response (protein loss and insulin resistance), contractile dysfunction, and disruption of muscle regenerative ability in many chronic disease states (20-24). Although, neutralization of some of these 
cytokines attenuates muscle wasting, blocking their activity failed to preserve skeletal muscle mass in some conditions where even their abundance was significantly elevated suggesting that there are additional mediators of muscle wasting (24). TNF-like weak inducer of apoptosis (TWEAK), a member of the TNF superfamily, has recently been identified as an important mediator of skeletal muscle wasting and metabolic dysfunction. While the levels of several classical muscle wasting cytokines are increased in various chronic disease states, there is no evidence about their potential role in the loss of muscle mass in disuse conditions (e.g., denervation, immobilization, and unloading). Our studies have shown that TWEAK/Fn14 system also mediates skeletal muscle wasting in disuse conditions and even in response to starvation. Moreover, increased levels of TWEAK inhibit skeletal muscle regenerative capacity through affecting self-renewal of satellite cells and proliferation, fusion, and differentiation of myoblasts into multinucleated myotubes both in vitro and in vivo (25-29). The role of TWEAK/Fn14 signaling in regulation of muscle progenitor cell biology has been discussed in another article in this issue (30). We have focused our review article to discuss the recent advancement toward understanding the role and mechanisms of action of TWEAK in adult skeletal muscle.

\section{BRIEF OVERVIEW TWEAK/Fn14 DYAD}

TWEAK is a member of TNF super family (TNFSF) cytokines, which is initially synthesized as type II transmembrane protein (249 amino acids) similar to TNF- $\alpha$. However, membrane-bound TWEAK is cleaved to its soluble form (156 amino acids) by furin, a calcium-dependent serine endoprotease (31-33). Fn14, a type I transmembrane protein, was first recognized by differential display analysis and later identified as the unique TWEAK receptor (34-36). Fn14 is the smallest member (102 amino acids) of TNF receptor super family (TNFRSF), which is expressed at relatively low levels in normal healthy tissues except in progenitor cells. However, the expression of Fn14 is drastically increased in response to tissue injury and various pathological conditions (31, 33). Similar to other members of TNFRSF, the cytoplasmic domain of Fn 14 contains a TRAF binding site, which allows downstream signaling upon stimulation by TWEAK (37). By contrast, Fn14 lacks a death domain and does not show any association with death-inducing signaling complex (33). Furthermore, some studies suggest that Fn14 can also function by clustering themselves on the cell membrane under low TWEAK condition. TWEAK/Fn14 system plays an important role in tissue homeostasis through regulating cell survival, proliferation, migration, and angiogenesis $(31,33)$. Conversely, aberrant regulation of TWEAK/Fn14 signaling causes many pathological consequences including cancer, rheumatoid arthritis, systemic lupus erythematous, multiple sclerosis, skeletal muscle wasting, and cardiac dysfunction and failure (31).

Like other members of TNFSF, TWEAK mediates unique and context-dependent pleiotropic effects. In contrast to TNF$\alpha$, TWEAK attenuates the transition from innate to adaptive immunity by suppressing the production of interferon- $\gamma$ and IL-12 cytokines (38). Moreover, TWEAK activates multiple intracellular pathways such as MAPK, PI3K/Akt, and canonical and non-canonical NF- $\mathrm{B}$ signaling in various cell types (3942). Binding of TWEAK leads to the formation of TRAF2/cIAP1 (cellular inhibitor of apoptosis protein 1) complex at Fn14 cytoplasmic domain that results in the activation of various signaling proteins including TRAF6, transforming growth factor- $\beta$ activated kinase1 (TAK1), I kappa $\beta$ kinase (IKK), NF-кB-inducing kinase (NIK), and MAPKs, which regulate expression of several molecules involved in various cellular responses (Figure 1) (33, $39,43-45)$. Moreover, the activation of TWEAK/Fn14 is coupled with TNF $\alpha-T N F R 1$ signaling. For example, the activation of TWEAK/Fn14 signaling sensitizes TNF- $\alpha$ signaling by exhausting cytosolic TRAF2/cIAP1 complex (44).

\section{TWEAK CAUSES ATROPHY IN CULTURED MYOTUBES}

$\mathrm{C} 2 \mathrm{C} 12$ as well as mouse primary myoblasts differentiate into myotubes upon incubation in low serum conditions. These cultured myotubes serve as an excellent model to study atrophy in response to exogenously added molecules. We have previously reported that treatment of cultured myotubes with even low concentrations of TWEAK protein causes severe atrophy $(46,47)$. Multiple studies have shown that skeletal muscle atrophy is associated with rapid degradation of selective muscle proteins including myosin heavy chain (MyHC) (48-50). Consistent with effects on myotube size, addition of TWEAK led to significant reduction in overall protein content and levels of $\mathrm{MyHC}$ in cultured myotubes $(46,47)$. Moreover, at equimolar concentration, TWEAK was found to be more potent in inducing MyHC degradation compared with its structural homolog TNF- $\alpha$ suggesting that TWEAK is a potent muscle wasting cytokine (47).

The UPS is one of the most important proteolytic systems that cause selective degradation of muscle structural proteins $(1,11$, 51). Two muscle-specific E3 ubiquitin ligases, muscle RING-finger $1(M u R F 1)$ and muscle atrophy F-box (MAFBx; also known as Atrogin-1) are the key enzymes of the UPS involved in degradation of muscle proteins. Levels of both MuRF1 and MAFBx are drastically increased in atrophying skeletal muscle, where disruption of these genes in mice preserves skeletal muscle mass in many catabolic conditions, such as hind limb unloading, cast immobilization, and denervation $(1,11,52)$. ALS is another proteolytic mechanism that contributes to degradation of muscle proteins and cellular organelles in skeletal muscle in response to various atrophic stimuli. In autophagy, cytosolic proteins and organelles are sequestered by an isolation-membrane structure to form autophagosome. The autophagosome then fuses with lysosome, where the autophagosome is degraded to amino acid or peptide (53). Autophagy constitutively takes place at lower levels in most cells under normal condition and is an essential component of homeostasis machinery in different tissues including skeletal muscle $(53,54)$. However, excessive activation of autophagy leads to muscle wasting in many conditions such as starvation and denervation $(8,55)$.

Our studies have shown that TWEAK increases the gene expression of MuRF1 and MAFBx and stimulates the conjugation of ubiquitin with MyHC in cultured myotubes $(46,47)$. Furthermore, TWEAK also induces the expression of several components of ALS and activates caspases in cultured myotubes (46). Indeed, inhibition of the activity of either UPS, autophagy, or caspases 


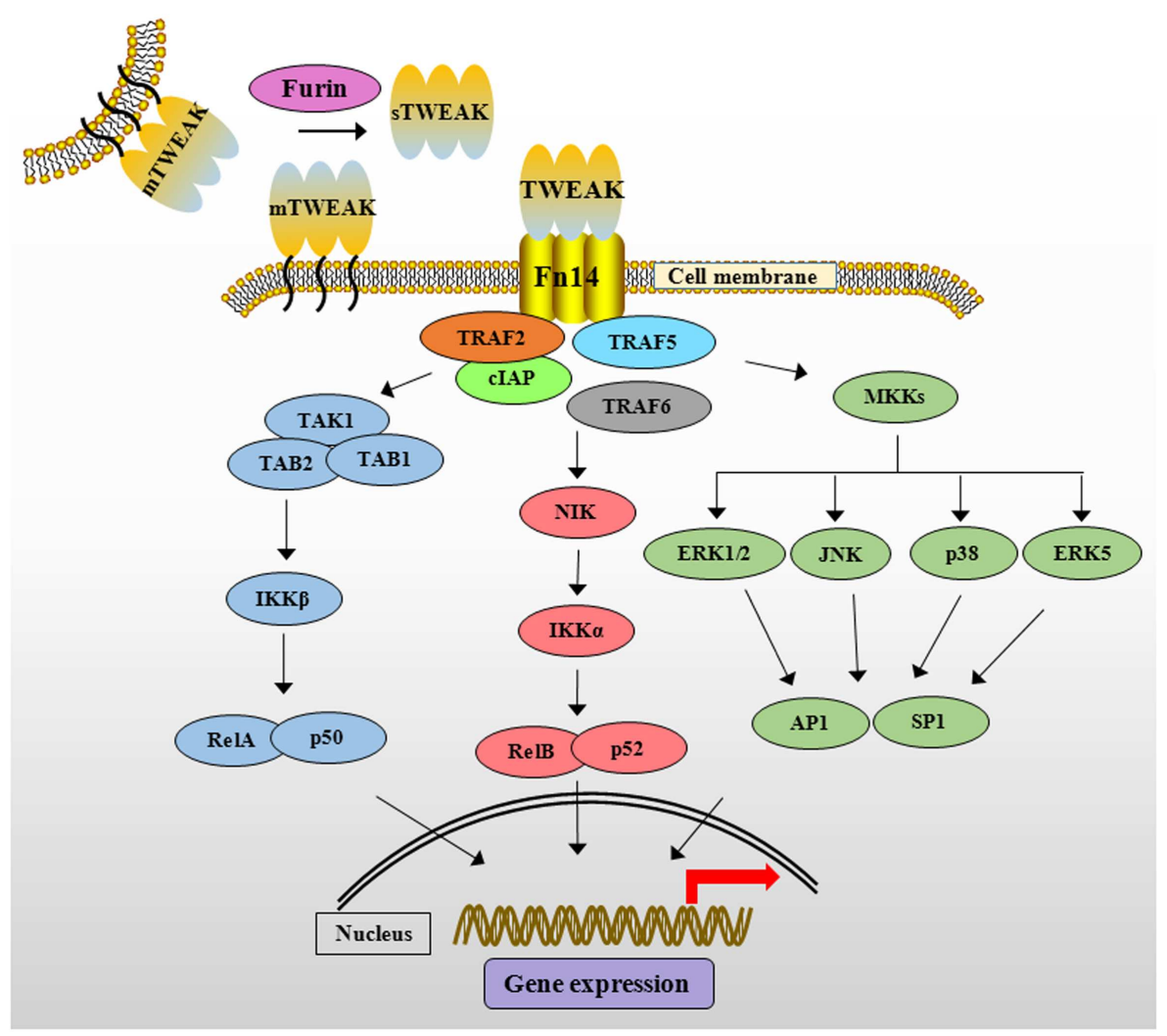

FIGURE 1 |The TWEAK/Fn14 signaling cascade. TWEAK cytokine is initially synthesized as membrane-anchored protein (mTWEAK). mTWEAK is then cleaved by furin into soluble form of TWEAK (sTWEAK). Binding of trimeric mTWEAK or sTWEAK to Fn14 receptor leads to the recruitment of clAPs and various TRAFs resulting in the activation of multiple downstream signaling cascades. TWEAK stimulates canonical NF-KB pathway through activation of TAK 1 and IKK $\beta$ and phosphorylation and degradation of $I_{\kappa} B$ protein. TWEAK activates non-canonical NF-אB pathway through activation of NIK and IKK $\alpha$ leading to proteolytic processing of p100 protein into p52. TWEAK-mediated signaling also causes the activation of various MKKs, which phosphorylate ERK1/2, ERK5, JNK, and p38MAPK resulting in the activation of downstream transcription factors such as AP1 and SP1. TWEAK, TNF-like weak inducer of apoptosis; Fn14, fibroblast growth factor inducible 14; clAP, cellular inhibitor of apoptosis; TRAF, TNF

receptor-associated factor; TAK1, transforming growth factor $\beta$-activated kinase 1, TAB, TAK1 binding protein; IKK, I $\mathrm{K}$ kinase $\beta$; $I_{\kappa} B$, inhibitor of nuclear factor of kappa B; NIK, NF-кB-inducing kinase; MAPK, mitogen-activated protein kinase; MKKs, mitogen-activated protein kinase kinases; ERK, extracellular signal-regulating kinase; JNK, c-Jun N-terminal kinase; AP1, activator protein 1; SP1, specificity protein 1. significantly reduced TWEAK-induced degradation of MyHC and myotube atrophy suggesting that TWEAK induces muscle proteolysis through coordinated activation of multiple proteolytic systems (46).

$\mathrm{NF}-\kappa \mathrm{B}$ is a major proinflammatory transcription factor, which is strongly linked to skeletal muscle wasting not only in chronic diseases but also in disuse conditions (10). Inhibition of NF- $\mathrm{B}$ has been found to rescue skeletal muscle atrophy in response to cytokines, tumor growth, denervation, and unloading $(10,56)$. By contrast, forced activation of NF- $\kappa \mathrm{B}$ is sufficient to cause severe muscle wasting in mice (56). NF- $\kappa \mathrm{B}$ induces muscle atrophy through augmenting the expression of MuRF1 and several other components of UPS (10). It has been recently reported that MuRF1 causes breakdown of $\mathrm{MyHC}$ and other components of the thick filament of the sarcomere during atrophy $(57,58)$. Our studies have shown that TWEAK causes sustained activation of NF- $\kappa$ B. Furthermore, inhibition of NF- $\kappa$ B through pharmacological or molecular approaches prevents the degradation of MyHC and atrophy in cultured myotubes (46, 47, 59, 60). Although,
TWEAK did not inhibit protein synthesis, it inhibited the activation of PI3K/Akt/mTOR signaling pathway in cultured myotubes (47). Similarly, TWEAK has also been found to inhibit the insulininduced Akt phosphorylation in hepatocytes (61). Since Akt signaling inhibits the activity of FoxO family of transcription factors, which are required for the expression of MuRF1 and MAFBx (1), it is likely that the inhibition of Akt signaling is another mechanism by which TWEAK augments the gene expression of MuRF1 and MAFBx in cultured myotubes. Collectively, these observations suggest that TWEAK causes atrophy in cultured myotubes through the activation of NF- $\kappa \mathrm{B}$ and various proteolytic systems such as UPS, ALS, and caspases (Figure 2).

The effect of TWEAK on global gene expression and microRNAs (miRs) in cultured myotubes has also been investigated. Pathway analysis of microarray gene expression data suggested that TWEAK affects the activation of several toxic pathways including those involved in initiation and manifestation of fibrosis, oxidative stress, and mitochondrial dysfunction in skeletal muscle (62). For example, TWEAK reduces the expression of structural molecules 


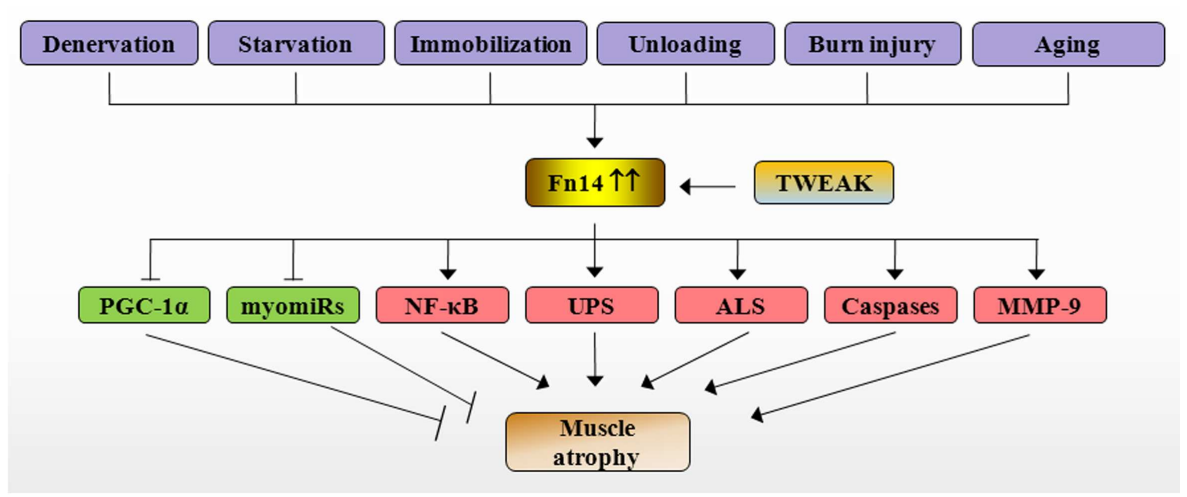

FIGURE 2 | Mechanisms of action of TWEAK/Fn14 system in skeletal muscle atrophy. Specific catabolic conditions such as denervation, starvation, immobilization, unloading, burn injury, and aging augments the expression of Fn14 resulting in stimulation of TWEAK/Fn14 signaling in skeletal muscle. TWEAK-Fn14 signaling represses the levels of PGC-1 $\alpha$ and myomiRs and increases the activation of NF- $\mathrm{B}$, UPS, ALS, caspases, and
MMP-9, which eventually cause loss of skeletal muscle mass. Fn14, fibroblast growth factor inducible 14; TWEAK, TNF-like weak inducer of apoptosis; PGC-1 $\alpha$, peroxisome proliferator-activated receptor-gamma coactivator $1 \alpha$; MMP-9, matrix metalloproteinase-9; NF-кB, nuclear factor-kappa B; UPS, ubiquitin-proteasome system; ALS, autophagy-lysosome system; myomiRs, muscle microRNAs. (e.g. MyHC, TCap, and ankyrin repeat domain 2), metabolic enzymes (e.g., phosphoglycerate mutase 2), and signaling proteins (e.g., Notch1 and TRAF6) in C2C12 myotubes. By contrast, TWEAK induces the expression of multiple components of NF- $\kappa$ B signaling pathways and expression of matrix metalloproteinase- 9 (MMP-9) in myotubes (62). Recently, a few muscle-specific miRs such as miR-1, miR-133a, miR-133b, and miR-206 (also called myomiRs) have been identified, which are essential for muscle progenitor cell proliferation, differentiation, and maintenance. Expression of miR-1 and miR-133a in embryonic stem cells and other non-muscle cell types showed that they promote the differentiation into the skeletal muscle lineage. Unlike other myomiRs, which are also expressed in cardiac tissues, miR-133b and miR-206 are predominantly expressed in skeletal muscle (63). Interestingly, microarray analysis of miRs has shown that TWEAK reduces the expression levels of miR-1, miR-133a, miR-133b, and miR-206 in cultured myotubes (62). Recent studies have demonstrated that myogenic transcription factors, such as myocyte enhancer factor $2 \mathrm{c}$ (MEF2c) and MyoD control the expression of myomiRs in skeletal and cardiac muscles (63). Interestingly, TWEAK diminishes the levels of both MEF2c and MyoD $(25,62)$ suggesting that reduced expression of myomiRs could be a result of diminished abundance of MEF2c and MyoD in TWEAK-treated myotubes. TWEAK also regulates the expression of a number of other miRs, which are involved in distinct biological responses. Using differentially regulated miR data set, we generated a network of pathways from differentially regulated genes in cDNA microarray data set. This analysis showed that miRs network overlaps with mRNA networks suggesting that miRs may play important roles in the regulation of gene expression and skeletal muscle structure and function in response to TWEAK (62).

\section{TWEAK CAUSES SKELETAL MUSCLE ATROPHY IN VIVO}

In vivo effects of TWEAK on skeletal muscle were first investigated by systemic and chronic administration of soluble TWEAK proteins in wild-type mice. Chronic administration of TWEAK in mice led to a significant reduction in whole body weight, individual muscle mass, and fiber-cross-sectional area (47). Furthermore, transgenic ( $\mathrm{Tg}$ ) overexpression of full-length TWEAK using muscle creatine kinase (MCK) promoter resulted in smaller muscle size and neonatal lethality in mice (47). TWEAK-Tg mice, which survived due to relatively low expression of TWEAK (four to sixfolds higher than littermate controls) showed muscle atrophy and interstitial fibrosis at around 4-6 months of age (43). Consistent with in vitro studies, transcript levels of MuRF1 and ubiquitination of MyHC were significantly elevated in skeletal muscle of TWEAKTg mice compared with their littermate controls. By contrast, there was no significant difference in mRNA levels of MAFBx in skeletal muscle of wild-type and TWEAK-Tg mice indicating that TWEAK causes muscle atrophy in vivo through augmenting the expression of MuRF1 $(43,62)$. Furthermore, the gene expression of several components of NF- $\kappa \mathrm{B}$ signaling pathway and DNA-binding activity of NF- $\kappa \mathrm{B}$ were significantly increased in skeletal muscle of TWEAK-Tg mice (43). These findings suggest that the activation of NF- $\kappa \mathrm{B}$ pathway is one of the important mechanisms for TWEAK-induced muscle proteolysis (Figure 2).

Accumulating evidence suggests that peroxisome proliferatoractivated receptor $\gamma($ PPAR- $\gamma$ ) coactivator $1 \alpha$ (PGC- $1 \alpha)$ plays a key role in preserving skeletal muscle mass and mitochondrial content in atrophic conditions (64). Levels of PGC-1 $\alpha$ are repressed in multiple atrophy conditions whereas muscle-specific overexpression of PGC- $1 \alpha$ rescues the loss of skeletal muscle mass in catabolic states $(65,66)$. Our recent studies demonstrate that TWEAK represses the expression of PGC-1 $\alpha$ in cultured myotubes (67). Overexpression of PGC- $1 \alpha$ inhibits TWEAK-induced atrophy, NF- $\kappa \mathrm{B}$ activation, and expression of MAFBx and MuRF1 in cultured myotubes. Moreover, progressive muscle atrophy observed in TWEAK-Tg mice is significantly attenuated in TWEAK-PGC$1 \alpha$ double Tg mice suggesting that PGC-1 $\alpha$ plays an important role in TWEAK-induced muscle atrophy (67).

Another potential mechanism by which TWEAK causes muscle atrophy is through deregulation of matrix metalloproteinases 
(MMPs). Protein levels as well as enzymatic activity of MMP-9 are significantly elevated in skeletal muscle of TWEAK-Tg mice (59, 62). Since, muscle atrophy in response to chronic administration of TWEAK is rescued to some extent in Mmp9-knockout (KO) mice, it is likely that MMP-9 is involved in TWEAK-induced skeletal muscle wasting in vivo. Elevated levels of MMP-9 can potentially induce muscle atrophy through breakdown of extracellular matrix and proteolytic activation of other catabolic cytokines (59). In agreement with microarray data in TWEAK-treated myotubes, we have found that the levels of many structural molecules such as $\mathrm{MyHC}$, TCap, and ankyrin repeat domain 2 and myomiRs were reduced in skeletal muscle of TWEAK-Tg mice suggesting that TWEAK also causes atrophy through repression of specific muscle proteins (62).

\section{TWEAK/Fn14 SYSTEM MEDIATES MUSCLE ATROPHY IN CATABOLIC CONDITIONS}

Muscle atrophy can be observed in various physiological and pathophysiological conditions such as bed-rest, space flight, aging, cancer, severe burn injury, starvation, high dose glucocorticoid therapy, and neurotmesis (2). Our group and others have recently investigated whether the gene expression of TWEAK or Fn14 is affected in skeletal muscle in different atrophy conditions. Interestingly, the gene expression of Fn14 is dramatically induced in skeletal muscle in disuse conditions such as denervation and immobilization (43) and in response to starvation (48). Wu et al. studied the global gene expression in skeletal muscle of mice in response to hind limb suspension, a model to elicit unloadinginduced skeletal muscle atrophy. Microarray and quantitative real-time PCR assays showed that the gene expression of Fn14 is significantly increased in skeletal muscle of mice (68). Moreover, a recent study has also demonstrated that the levels of TWEAK and Fn14 are increased in human skeletal muscle in response to severe burn injury (69). By contrast, mRNA levels of TWEAK and Fn14 remained unaffected in skeletal muscle of mice in response to high doses of glucocorticoids (43) and in skeletal muscle of patients with chronic obstructive pulmonary disease (70) indicating that TWEAK/Fn14 system may not be the mediator of muscle atrophy in all conditions.

The physiological role of TWEAK/Fn14 system in muscle atrophy has been investigated through a series of experiments using denervated hind limb muscle as a model of neurotmesis (43). Levels of Fn14 protein are dramatically increased upon denervation regardless of muscle fiber type (43). Denervation-induced loss of skeletal muscle mass and fiber cross-sectional-area were significantly exaggerated in TWEAK-Tg mice but ameliorated in TWEAK-KO mice compared with wild-type mice. Denervated soleus muscle of TWEAK-KO mice showed improved absolute force compared with corresponding wild-type mice. Skeletal muscle of TWEAK-Tg mice also showed increased gene expression of collagen I and collagen III and interstitial fibrosis upon denervation indicating that TWEAK can directly induce fibrosis in skeletal muscle tissues. Similar to cell culture studies, TWEAK stimulated the DNA-binding activity of NF- $\mathrm{B}$ and increased the gene expression of MuRF1 in denervated skeletal muscle (43). Furthermore, starvation-induced loss of skeletal muscle mass was reduced in TWEAK-KO mice compared with wild-type mice (43). Collectively, these studies provide strong evidence that TWEAK/Fn14 system mediates muscle atrophy in catabolic conditions (Figure 2). It is noteworthy that denervation and starvation did not affect the expression TWEAK in skeletal muscle of mice. Similar to studies in rodents, a recent study reported that chronic hemiplegia-induced muscle atrophy was not accompanied with changes in expression of TWEAK in humans (71).

While TWEAK affects the activation of a number of signaling pathways and proteolytic systems in cultured myotubes, only a few of them appear to be regulated by TWEAK in skeletal muscle of mice in atrophic conditions. For example, TWEAK inhibits the activity of PI3K/Akt pathway in cultured myotubes; however, there was no difference in level of phosphorylation of Akt in denervated muscle of wild-type, TWEAK-Tg and TWEAKKO mice. Furthermore, while denervation increased expression of several autophagy-related genes (such as LC3B, Beclin1, Atg5, Atg12, and Gabarapl1), their expression levels were comparable in denervated skeletal muscle of wild-type, TWEAK-Tg and TWEAK-KO mice (43). These results suggest that denervated skeletal muscle is enriched with certain growth factors, which neutralize some of the actions of TWEAK. However, we cannot rule out that TWEAK/Fn14 signaling also affects the activation of PI3K/Akt and ALS in other conditions of muscle atrophy.

\section{REGULATION OF Fn14 EXPRESSION IN SKELETAL MUSCLE}

While, it is increasingly clear that the expression of Fn14 is a rate-limiting step, molecular mechanisms leading to its increased expression in skeletal muscle in atrophic conditions remain poorly understood. Both human and mouse Fn 14 promoters lack a typical TATA box, which is generally required for the expression of mammalian genes. However, the promoter region of Fn14 contains consensus sequence for several transcription factors (72). Recent studies in our laboratory have shown that mouse and human Fn14 promoters contain a cytosine-guanine dinucleotide (CpG) island close to transcription start site. Fn14 promoter also contains consensus DNA sequence for AP1 and specificity protein 1 (SP1) transcription factors. Our studies suggest that denervation causes hypomethylation at specific $\mathrm{CpG}$ sites in mouse Fn14 promoter in skeletal muscle. Interestingly, while all three DNA methyltransferases (Dnmts) interact with Fn14 promoter in naïve muscle, the levels of DNA methyltransferase 3a (Dnmt3a) are repressed in denervated skeletal muscle of mice. The role of Dnmt3a in Fn14 expression has been supported by our findings that overexpression of Dnmt3a inhibits the expression of Fn14 and attenuates denervation-induced muscle atrophy. We have also found that denervation increases the activation of MAPK, AP1, and SP1 and they are involved in the expression of Fn14 in denervated skeletal muscle.

\section{TWEAK PROMOTES SLOW-TO-FAST FIBER TYPE TRANSITION IN SKELETAL MUSCLE}

Rodent skeletal muscle contains four fiber types based on the primary expression of MyHC: I, IIA, IIX, and IIB (73, 74). Type I fibers are slow to twitch by stimuli, display a two to threefold higher mitochondrial content, and rely largely on oxidative metabolism to produce ATP. In contrast, type II fibers are fast 
in responding to stimuli and rely on glycolytic metabolism as a major energy substrate (75). Type IIB fibers have relatively few mitochondria and store large amounts of glycogen; therefore they are glycolytic in metabolism. Types IIA and IIX fibers have physiologically intermediate properties of slow and fast twitch fibers, which are also rich in mitochondria and possess a relatively high capacity to generate ATP by oxidative metabolism $(76,77)$. Moreover, it is now increasingly evident that different fiber types display different sensitivity to atrophy stimuli. Oxidative fibers are somewhat resilient to atrophy upon denervation (78) whereas glycolytic fibers undergo more rapid atrophy in response to starvation or sepsis $(79,80)$.

TWEAK/Fn14 axis appears to be an important regulator of fiber type composition in skeletal muscle. Tg overexpression of TWEAK in mice causes a significant reduction in proportion of type I fibers with a concomitant increase in type II fibers in both soleus and extensor digitorum longus muscle (43). Moreover, hind limb muscle of the founder TWEAK-Tg mice, which could not survive beyond neonatal age, appeared pale compared with wild-type littermates indicating lower myoglobin abundance and fast muscle phenotype in TWEAK overexpressing muscle (47). By contrast, proportion of types I and IIA muscle fibers and skeletal muscle mitochondrial content was found to be increased in skeletal muscle of TWEAK-KO mice $(43,81)$. These results suggest that TWEAK favors fast-type fiber phenotype even under normal conditions.

Recent studies have shown that PGC-1 $\alpha$ plays an important role in regulating skeletal muscle fiber composition, mitochondrial content, and oxidative metabolism in both physiological and pathophysiological conditions $(64,82,83)$. PGC- $1 \alpha$ is expressed preferentially in skeletal muscle enriched in type I fibers. Positive role of PGC- $1 \alpha$ in inducing type I fibers is supported by the findings that Tg overexpression of PGC- $1 \alpha$ in skeletal muscle increases the proportion of types I and IIA fibers (84). Conversely, muscle-specific PGC- $1 \alpha$ KO mice exhibited a shift from oxidative types I and IIA toward types IIX and IIB muscle fibers (85) while global PGC-1 $\alpha$ KO mice did not show a marked skeletal muscle phenotype $(85,86)$. PGC- $1 \alpha$ drives the transcription of type I muscle fibers through co-activation of myocyte enhancer factor 2 (MEF2) family members such as MEF2c and MEF2d (84). MEF2 and PGC-1 $\alpha$ also regulate the expression of PGC- $1 \alpha$ in an auto-regulatory loop (87). Interestingly, our recent studies have shown that TWEAK suppresses the expression of MEF2c in $\mathrm{C} 2 \mathrm{C} 12$ myotubes (62). Therefore, it is likely that TWEAK increases the number of type II fibers through reducing MEF2/PGC-1 $\alpha$ signaling (Figure 3). Supporting this notion, our recent study has demonstrated that transcript levels of PGC- $1 \alpha$ and several molecules involved in mitochondrial oxidative metabolism are significantly increased in skeletal muscle of 5-month-old TWEAKKO mice. Furthermore, treatment of cultured primary myotubes with TWEAK drastically reduced levels of PGC- $1 \alpha$ and other mitochondria-related genes (81). Likewise, an inverse relationship between TWEAK and PGC- $1 \alpha$ has been observed in denervated skeletal muscle of mice (67). Since type II muscle fibers are more susceptible to atrophy compared to type I muscle fibers in many chronic diseases, it will be interesting to investigate whether slow- to fast-type fiber transition is essential for TWEAK-induced muscle atrophy.

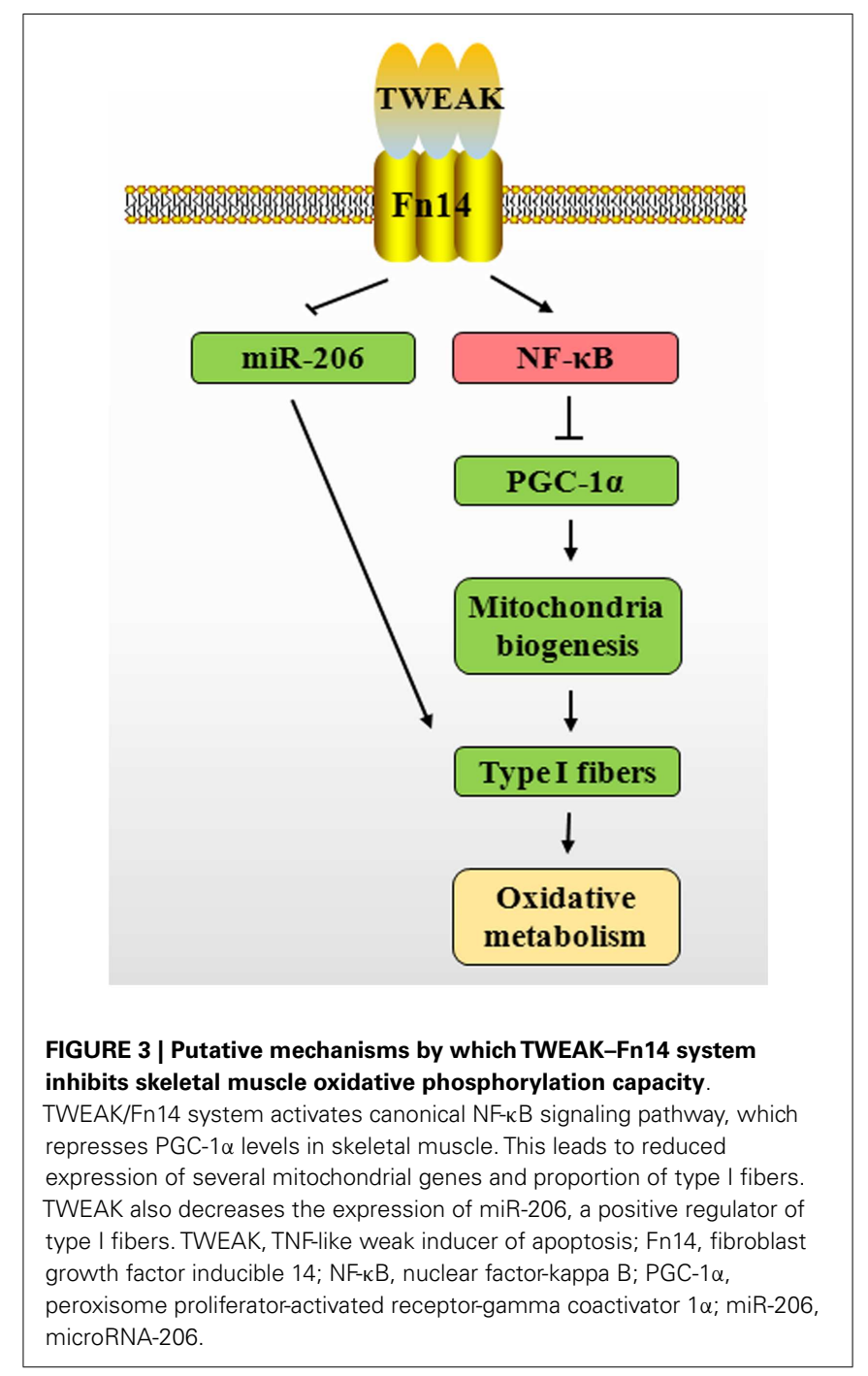

\section{TWEAK INHIBITS SKELETAL MUSCLE OXIDATIVE METABOLISM}

Skeletal muscle, due to its large mass, is the principal organ for glucose disposal in the body and therefore even a small reduction in skeletal muscle glucose uptake capacity or its ability to metabolize glucose is sufficient to cause metabolic abnormalities and obesity. The decline in skeletal muscle oxidative phosphorylation capacity during aging has been recognized as a major cause for increased fatigability, lowered quality of life, and morbidity (19). The loss of oxidative capacity with elevated levels of proinflammatory cytokines is also implicated in development of metabolic syndrome in many chronic disease states, while the maintenance with exercise has been found to be beneficial to some extent $(88,89)$.

We have recently studied the role of TWEAK in exercise tolerance and skeletal muscle oxidative phosphorylation capacity. TWEAK-KO mice run longer and with higher speed in a treadmill exercise tolerance test (81). Furthermore, TWEAK-KO mice show augmented levels of subsarcolemmal and intermyofibrillar mitochondria, increased succinate dehydrogenase (SDH)-positive 
myofibers, and elevated gene expression of metabolic proteins such as PGC- $1 \alpha, \operatorname{PPAR} \delta$, and mCPT-1. Moreover, oxidative phosphorylation is also increased in exercised TWEAK-KO mice compared with wild-type mice. Consistent with studies in TWEAK-KO mice, treatment of cultured myotubes with TWEAK decreased mitochondrial biogenetic capacity and maximal respiratory activity. TWEAK also reduced the expression of PGC- $1 \alpha$ and several mitochondrial genes in cultured myotubes (81). Recent studies also suggest that TWEAK represses PGC- $1 \alpha$ in cultured cardiomyocytes and myotubes through the activation of canonical NF- $\kappa \mathrm{B}$ signaling pathway $(67,90)$. Collectively, these studies suggest that the repression of PGC- $1 \alpha$ is an important mechanism by which TWEAK reduces mitochondrial content and oxidative phosphorylation capacity in skeletal muscle and in other cell types.

There is also a possibility that TWEAK inhibits skeletal muscle oxidative phosphorylation capacity through affecting levels of various miRs. The expression of miRs is sensitive to cytokine levels and alternation of miRs in response to inappropriate cytokine simulation may result in disrupting metabolic homeostasis (91). A subset of muscle-specific miRs has been shown to play an important role in skeletal muscle development and metabolic adaptation (92). Abundance of miR-206 is significantly higher in slow-type skeletal muscle compared with fast-type muscle (93). Levels of miR-206 are diminished during slow-to-fast fiber type transition in response to unloading (94). Furthermore, levels of miR-206 in vastus lateralis have been found to be significantly decreased in patients with type II diabetes compared with healthy individuals (95). Our low-density miRNA array analysis has shown that TWEAK inhibits the expression of miR-206 in C2C12 myotubes (62). These findings suggest that TWEAK may also inhibit skeletal muscle oxidative metabolism through down-regulation of miR-206 levels (Figure 3).

\section{CONCLUDING REMARKS}

The studies summarized above indicate that TWEAK/Fn14 system plays an essential role in skeletal muscle remodeling and metabolism. Most of these observations suggest that TWEAK/Fn14 signaling causes loss of skeletal muscle mass and decreases skeletal muscle oxidative metabolism implying that the inhibition of this cytokine-receptor axis can be used as a therapy to maintain skeletal muscle mass and metabolic function. We believe that TWEAK/Fn14 system is among the most attractive drug targets to combat muscle wasting. TWEAK being an extracellular protein per se, TWEAK-dependent signaling can be blocked using a TWEAK neutralizing antibody or soluble Fn14-Fc decoy protein, which prevents TWEAK binding to Fn14 cell surface receptors. Indeed, these two reagents have been found to be effective in improving pathologic condition in mouse models of some other diseases where TWEAK/Fn14 signaling is elevated. Alternatively, small-molecule antagonists that prevent Fn14 trimerization or interaction of TWEAK with Fn14 can also be used to block catabolic actions of TWEAK in skeletal muscle.

Whereas the role of TWEAK/Fn14 signaling in skeletal muscle has become increasingly clear, there are still some outstanding questions that need to be addressed. For example, it is important to identify other conditions where TWEAK/Fn14 axis is a mediator of muscle wasting. Furthermore, it is important to investigate the mechanism by which TWEAK causes metabolic abnormalities and whether the inhibition of TWEAK/Fn14 signaling can prevent type II diabetes and obesity in response to high fat diet. It will also be interesting to examine whether TWEAK affects mitochondrial content through regulating mitochondria biogenesis, fusion, fission, or mitophagy. The role of various miRs in regulation of TWEAK-induced muscle atrophy and metabolic dysfunction also needs more investigation. Nevertheless, recent studies have provided strong evidence that TWEAK/Fn14 system is a major regulator of skeletal muscle mass and function.

\section{AUTHOR CONTRIBUTIONS}

Ashok Kumar conceptualized the study. Shuichi Sato, Yuji Ogura, and Ashok Kumar wrote the manuscript.

\section{ACKNOWLEDGMENTS}

We are grateful to Ms. Sajedah M. Hindi for her critical reading of this manuscript. This work was supported by National Institute of Health grants AG029623 and AR059810 to Ashok Kumar.

\section{REFERENCES}

1. Bonaldo P, Sandri M. Cellular and molecular mechanisms of muscle atrophy. Dis Model Mech (2013) 6(1):25-39. doi:10.1242/dmm.010389

2. Jackman RW, Kandarian SC. The molecular basis of skeletal muscle atrophy. Am J Physiol Cell Physiol (2004) 287(4):C834-43. doi:10.1152/ajpcell.00579. 2003

3. Glass DJ. Signaling pathways perturbing muscle mass. Curr Opin Clin Nutr Metab Care (2010) 13(3):225-9. doi:10.1097/MCO.0b013e32833862df

4. Lecker SH, Jagoe RT, Gilbert A, Gomes M, Baracos V, Bailey J, et al. Multiple types of skeletal muscle atrophy involve a common program of changes in gene expression. FASEB J (2004) 18(1):39-51. doi:10.1096/fj.03-0610com

5. Kimball SR, Jefferson LS. Control of translation initiation through integration of signals generated by hormones, nutrients, and exercise. J Biol Chem (2010) 285(38):29027-32. doi:10.1074/jbc.R110.137208

6. Bodine SC, Stitt TN, Gonzalez M, Kline WO, Stover GL, Bauerlein R, et al. Akt/mTOR pathway is a crucial regulator of skeletal muscle hypertrophy and can prevent muscle atrophy in vivo. Nat Cell Biol (2001) 3(11):1014-9. doi:10.1038/ncb1101-1014

7. Rommel C, Bodine SC, Clarke BA, Rossman R, Nunez L, Stitt TN, et al. Mediation of IGF-1-induced skeletal myotube hypertrophy by PI(3)K/Akt/mTOR and PI(3)K/Akt/GSK3 pathways. Nat Cell Biol (2001) 3(11):1009-13. doi:10.1038/ ncb1101-1009

8. Sandri M, Sandri C, Gilbert A, Skurk C, Calabria E, Picard A, et al. Foxo transcription factors induce the atrophy-related ubiquitin ligase atrogin-1 and cause skeletal muscle atrophy. Cell (2004) 117(3):399-412. doi:10.1016/S00928674(04)00400-3

9. Latres E, Amini AR, Amini AA, Griffiths J, Martin FJ, Wei Y, et al. Insulin-like growth factor-1 (IGF-1) inversely regulates atrophy-induced genes via the phosphatidylinositol 3-kinase/Akt/mammalian target of rapamycin (PI3K/Akt/mTOR) pathway. J Biol Chem (2005) 280(4):2737-44. doi:10.1074/ jbc.M407517200

10. Li H, Malhotra S, Kumar A. Nuclear factor-kappa B signaling in skeletal muscle atrophy. J Mol Med (2008) 86(10):1113-26. doi:10.1007/s00109-0080373-8

11. Sandri M. Protein breakdown in muscle wasting: role of autophagy-lysosome and ubiquitin-proteasome. Int J Biochem Cell Biol (2013) 45(10):2121-9. doi:10.1016/j.biocel.2013.04.023

12. Bongers KS, Fox DK, Ebert SM, Kunkel SD, Dyle MC, Bullard SA, et al. Skeletal muscle denervation causes skeletal muscle atrophy through a pathway that involves both Gadd45a and HDAC4. Am J Physiol Endocrinol Metab (2013) 305(7):E907-15. doi:10.1152/ajpendo.00380.2013

13. Ebert SM, Dyle MC, Kunkel SD, Bullard SA, Bongers KS, Fox DK, et al. Stressinduced skeletal muscle Gadd45a expression reprograms myonuclei and causes 
muscle atrophy. J Biol Chem (2012) 287(33):27290-301. doi:10.1074/jbc.M112. 374777

14. Moresi V, Williams AH, Meadows E, Flynn JM, Potthoff MJ, McAnally J, et al. Myogenin and class II HDACs control neurogenic muscle atrophy by inducing E3 ubiquitin ligases. Cell (2010) 143(1):35-45. doi:10.1016/j.cell.2010. 09.004

15. Romanello V, Guadagnin E, Gomes L, Roder I, Sandri C, Petersen Y, et al. Mitochondrial fission and remodelling contributes to muscle atrophy. EMBO J (2010) 29(10):1774-85. doi:10.1038/emboj.2010.60

16. Lokireddy S, Wijesoma IW, Teng S, Bonala S, Gluckman PD, McFarlane C, et al. The ubiquitin ligase Mull induces mitophagy in skeletal muscle in response to muscle-wasting stimuli. Cell Metab (2012) 16(5):613-24. doi:10.1016/j.cmet. 2012.10.005

17. Holloszy JO. Biochemical adaptations in muscle. Effects of exercise on mitochondrial oxygen uptake and respiratory enzyme activity in skeletal muscle. J Biol Chem (1967) 242(9):2278-82.

18. Lanza IR, Sreekumaran Nair K. Regulation of skeletal muscle mitochondrial function: genes to proteins. Acta Physiol (2010) 199(4):529-47. doi:10.1111/j. 1748- 1716.2010.02124.x

19. Peterson CM, Johannsen DL, Ravussin E. Skeletal muscle mitochondria and aging: a review. J Aging Res (2012) 2012:194821. doi:10.1155/2012/194821

20. Durham WJ, Dillon EL, Sheffield-Moore M. Inflammatory burden and amino acid metabolism in cancer cachexia. Curr Opin Clin Nutr Metab Care (2009) 12(1):72-7. doi:10.1097/MCO.0b013e32831cef61

21. Langen RC, Gosker HR, Remels AH, Schols AM. Triggers and mechanisms of skeletal muscle wasting in chronic obstructive pulmonary disease. Int J Biochem Cell Biol (2013) 45(10):2245-56. doi:10.1016/j.biocel.2013.06.015

22. Bhatnagar S, Kumar A. The TWEAK-Fn14 system: breaking the silence of cytokine-induced skeletal muscle wasting. Curr Mol Med (2012) 12(1):3-13. doi:10.2174/156652412798376107

23. Argiles JM, Busquets S, Toledo M, Lopez-Soriano FJ. The role of cytokines in cancer cachexia. Curr Opin Support Palliat Care (2009) 3(4):263-8. doi:10.1097/ SPC.0b013e3283311d09

24. Spate U, Schulze PC. Proinflammatory cytokines and skeletal muscle. Curr Opin Clin Nutr Metab Care (2004) 7(3):265-9. doi:10.1097/00075197-20040500000005

25. Dogra C, Changotra H, Mohan S, Kumar A. Tumor necrosis factor-like weak inducer of apoptosis inhibits skeletal myogenesis through sustained activation of nuclear factor-kappaB and degradation of MyoD protein. J Biol Chem (2006) 281(15):10327-36. doi:10.1074/jbc.M511131200

26. Dogra C, Changotra H, Wergedal JE, Kumar A. Regulation of phosphatidylinositol 3-kinase (PI3K)/Akt and nuclear factor-kappa B signaling pathways in dystrophin-deficient skeletal muscle in response to mechanical stretch. J Cell Physiol (2006) 208(3):575-85. doi:10.1002/jcp.20696

27. Enwere EK, Holbrook J, Lejmi-Mrad R, Vineham J, Timusk K, Sivaraj B, et al. TWEAK and cIAP1 regulate myoblast fusion through the noncanonical NFkappaB signaling pathway. Sci Signal (2012) 5(246):ra75. doi:10.1126/scisignal. 2003086

28. Girgenrath M, Weng S, Kostek CA, Browning B, Wang M, Brown SA, et al. TWEAK, via its receptor Fn14, is a novel regulator of mesenchymal progenitor cells and skeletal muscle regeneration. EMBO J (2006) 25(24):5826-39. doi:10.1038/sj.emboj.7601441

29. Ogura Y, Mishra V, Hindi SM, Kuang S, Kumar A. Proinflammatory cytokine tumor necrosis factor (TNF)-like weak inducer of apoptosis (TWEAK) suppresses satellite cell self-renewal through inversely modulating Notch and NFkappaB signaling pathways. J Biol Chem (2013) 288(49):35159-69. doi:10.1074/ jbc.M113.517300

30. Enwere EK, Adam N, LaCasse EC, Korneluk RG. Role of the TWEAK-Fn14cIAP1-NF- $\mathrm{B}$ signaling axis in the regulation of myogenesis and muscle homeostasis. Front Immunol (2014) 5:34. doi:10.3389/fimmu.2014.00034

31. Burkly LC, Michaelson JS, Zheng TS. TWEAK/Fn14 pathway: an immunological switch for shaping tissue responses. Immunol Rev (2011) 244(1):99-114. doi:10.1111/j.1600-065X.2011.01054.X

32. Chicheportiche Y, Bourdon PR, Xu H, Hsu YM, Scott H, Hession C, et al. TWEAK, a new secreted ligand in the tumor necrosis factor family that weakly induces apoptosis. J Biol Chem (1997) 272(51):32401-10. doi:10.1074/jbc.272. 51.32401

33. Winkles JA. The TWEAK-Fn14 cytokine-receptor axis: discovery, biology and therapeutic targeting. Nat Rev Drug Discov (2008) 7(5):411-25. doi:10.1038/ $\operatorname{nrd} 2488$
34. Meighan-Mantha RL, Hsu DK, Guo Y, Brown SA, Feng SL, Peifley KA, et al. The mitogen-inducible Fn14 gene encodes a type I transmembrane protein that modulates fibroblast adhesion and migration. J Biol Chem (1999) 274(46):33166-76. doi:10.1074/jbc.274.46.33166

35. Winkles JA, Tran NL, Brown SA, Stains N, Cunliffe HE, Berens ME. Role of TWEAK and Fn14 in tumor biology. Front Biosci (2007) 12:2761-71. doi: $10.2741 / 2270$

36. Wiley SR, Cassiano L, Lofton T, Davis-Smith T, Winkles JA, Lindner V, et al. A novel TNF receptor family member binds TWEAK and is implicated in angiogenesis. Immunity (2001) 15(5):837-46. doi:10.1016/S1074-7613(01) 00232-1

37. Brown SA, Richards CM, Hanscom HN, Feng SL, Winkles JA. The Fn14 cytoplasmic tail binds tumour-necrosis-factor-receptor-associated factors 1, 2, 3 and 5 and mediates nuclear factor-kappaB activation. Biochem $J$ (2003) 371 (Pt 2):395-403. doi:10.1042/BJ20021730

38. Maecker H, Varfolomeev E, Kischkel F, Lawrence D, LeBlanc H, Lee W, et al. TWEAK attenuates the transition from innate to adaptive immunity. Cell (2005) 123(5):931-44. doi:10.1016/j.cell.2005.09.022

39. Srivastava AK, Qin X, Wedhas N, Arnush M, Linkhart TA, Chadwick RB, et al. Tumor necrosis factor-alpha augments matrix metalloproteinase-9 production in skeletal muscle cells through the activation of transforming growth factor-beta-activated kinase 1 (TAK1)-dependent signaling pathway. J Biol Chem (2007) 282(48):35113-24. doi:10.1074/jbc.M705329200

40. Han S, Yoon K, Lee K, Kim K, Jang H, Lee NK, et al. TNF-related weak inducer of apoptosis receptor, a TNF receptor superfamily member, activates NF-kappa B through TNF receptor-associated factors. Biochem Biophys Res Commun (2003) 305(4):789-96. doi:10.1016/S0006-291X(03)00852-0

41. Polek TC, Talpaz M, Darnay BG, Spivak-Kroizman T. TWEAK mediates signal transduction and differentiation of RAW264.7 cells in the absence of Fn14/TweakR. Evidence for a second TWEAK receptor. J Biol Chem (2003) 278(34):32317-23. doi:10.1074/jbc.M302518200

42. Dogra C, Hall SL, Wedhas N, Linkhart TA, Kumar A. Fibroblast growth factor inducible 14 (Fn14) is required for the expression of myogenic regulatory factors and differentiation of myoblasts into myotubes. Evidence for TWEAK-independent functions of Fn14 during myogenesis. J Biol Chem (2007) 282(20):15000-10. doi:10.1074/jbc.M608668200

43. Mittal A, Bhatnagar S, Kumar A, Lach-Trifilieff E, Wauters S, Li H, et al. The TWEAK-Fn14 system is a critical regulator of denervation-induced skeletal muscle atrophy in mice. J Cell Biol (2010) 188(6):833-49. doi:10.1083/jcb. 200909117

44. Vince JE, Chau D, Callus B, Wong WW, Hawkins CJ, Schneider P, et al. TWEAK-FN14 signaling induces lysosomal degradation of a cIAP1-TRAF2 complex to sensitize tumor cells to TNFalpha. J Cell Biol (2008) 182(1):171-84. doi:10.1083/jcb.200801010

45. Kumar M, Makonchuk DY, Li H, Mittal A, Kumar A. TNF-like weak inducer of apoptosis (TWEAK) activates proinflammatory signaling pathways and gene expression through the activation of TGF-beta-activated kinase 1. J Immunol (2009) 182(4):2439-48. doi:10.4049/jimmunol.0803357

46. Bhatnagar S, Mittal A, Gupta SK, Kumar A. TWEAK causes myotube atrophy through coordinated activation of ubiquitin-proteasome system, autophagy, and caspases. J Cell Physiol (2012) 227(3):1042-51. doi:10.1002/jcp.22821

47. Dogra C, Changotra H, Wedhas N, Qin X, Wergedal JE, Kumar A. TNFrelated weak inducer of apoptosis (TWEAK) is a potent skeletal muscle-wasting cytokine. FASEB J (2007) 21(8):1857-69. doi:10.1096/fj.06-7537com

48. Paul PK, Bhatnagar S, Mishra V, Srivastava S, Darnay BG, Choi Y, et al. The E3 ubiquitin ligase TRAF6 intercedes in starvation-induced skeletal muscle atrophy through multiple mechanisms. Mol Cell Biol (2012) 32(7):1248-59. doi:10.1128/MCB.06351-11

49. Paul PK, Gupta SK, Bhatnagar S, Panguluri SK, Darnay BG, Choi Y, et al. Targeted ablation of TRAF6 inhibits skeletal muscle wasting in mice. J Cell Biol (2010) 191(7):1395-411. doi:10.1083/jcb.201006098

50. Acharyya S, Ladner KJ, Nelsen LL, Damrauer J, Reiser PJ, Swoap S, et al. Cancer cachexia is regulated by selective targeting of skeletal muscle gene products. J Clin Invest (2004) 114(3):370-8. doi:10.1172/JCI20174

51. Solomon V, Goldberg AL. Importance of the ATP-ubiquitin-proteasome pathway in the degradation of soluble and myofibrillar proteins in rabbit muscle extracts. J Biol Chem (1996) 271(43):26690-7. doi:10.1074/jbc.271.43.26690

52. Bodine SC, Latres E, Baumhueter S, Lai VK, Nunez L, Clarke BA, et al. Identification of ubiquitin ligases required for skeletal muscle atrophy. Science (2001) 294(5547):1704-8. doi:10.1126/science.1065874 
53. Sandri M. Autophagy in skeletal muscle. FEBS Lett (2010) 584(7):1411-6. doi:10.1016/j.febslet.2010.01.056

54. Masiero E, Agatea L, Mammucari C, Blaauw B, Loro E, Komatsu M, et al. Autophagy is required to maintain muscle mass. Cell Metab (2009) 10(6):507-15. doi:10.1016/j.cmet.2009.10.008

55. Zhao J, Brault JJ, Schild A, Cao P, Sandri M, Schiaffino S, et al. FoxO3 coordinately activates protein degradation by the autophagic/lysosomal and proteasomal pathways in atrophying muscle cells. Cell Metab (2007) 6(6):472-83. doi:10.1016/j.cmet.2007.11.004

56. Cai D, Frantz JD, Tawa NE Jr, Melendez PA, Oh BC, Lidov HG, et al. IKKbeta/NF-kappaB activation causes severe muscle wasting in mice. Cell (2004) 119(2):285-98. doi:10.1016/j.cell.2004.09.027

57. Clarke BA, Drujan D, Willis MS, Murphy LO, Corpina RA, Burova E, et al. The E3 Ligase MuRF1 degrades myosin heavy chain protein in dexamethasone-treated skeletal muscle. Cell Metab (2007) 6(5):376-85. doi:10.1016/j.cmet.2007.09.009

58. Cohen S, Brault JJ, Gygi SP, Glass DJ, Valenzuela DM, Gartner C, et al. During muscle atrophy, thick, but not thin, filament components are degraded by MuRF1-dependent ubiquitylation. J Cell Biol (2009) 185(6):1083-95. doi:10. 1083/jcb.200901052

59. Li H, Mittal A, Paul PK, Kumar M, Srivastava DS, Tyagi SC, et al. Tumor necrosis factor-related weak inducer of apoptosis augments matrix metalloproteinase 9 (MMP-9) production in skeletal muscle through the activation of nuclear factor-kappaB-inducing kinase and p38 mitogen-activated protein kinase: a potential role of MMP-9 in myopathy. J Biol Chem (2009) 284(7):4439-50. doi:10.1074/jbc.M805546200

60. Yamaki T, Wu CL, Gustin M, Lim J, Jackman RW, Kandarian SC. Rel A/p65 is required for cytokine-induced myotube atrophy. Am J Physiol Cell Physiol (2012) 303(2):C135-42. doi:10.1152/ajpcell.00111.2012

61. Feng F, Wang L, Albanese N, Holmes A, Xia P. Tumor necrosis factor-like weak inducer of apoptosis attenuates the action of insulin in hepatocytes. Endocrinology (2008) 149(4):1505-13. doi:10.1210/en.2007-1119

62. Panguluri SK, Bhatnagar S, Kumar A, McCarthy JJ, Srivastava AK, Cooper NG, et al. Genomic profiling of messenger RNAs and microRNAs reveals potential mechanisms of TWEAK-induced skeletal muscle wasting in mice. PLoS One (2010) 5(1):e8760. doi:10.1371/journal.pone.0008760

63. van Rooij E, Liu N, Olson EN. MicroRNAs flex their muscles. Trends Genet (2008) 24(4):159-66. doi:10.1016/j.tig.2008.01.007

64. Arany Z. PGC-1 coactivators and skeletal muscle adaptations in health and disease. Curr Opin Genet Dev (2008) 18(5):426-34. doi:10.1016/j.gde.2008.07.018

65. Brault JJ, Jespersen JG, Goldberg AL. Peroxisome proliferator-activated receptor gamma coactivator lalpha or lbeta overexpression inhibits muscle protein degradation, induction of ubiquitin ligases, and disuse atrophy. J Biol Chem (2011) 285(25):19460-71. doi:10.1074/jbc.M110.113092

66. Sandri M, Lin J, Handschin C, Yang W, Arany ZP, Lecker SH, et al. PGC-1alpha protects skeletal muscle from atrophy by suppressing FoxO3 action and atrophyspecific gene transcription. Proc Natl Acad Sci U S A (2006) 103(44):16260-5. doi:10.1073/pnas.0607795103

67. Hindi SM, Mishra V, Bhatnagar S, Tajrishi MM, Ogura Y, Yan Z, et al. Regulatory circuitry of TWEAK-Fn14 system and PGC-1alpha in skeletal muscle atrophy program. FASEB J (2013). doi:10.1096/fj.13-242123

68. Wu CL, Kandarian SC, Jackman RW. Identification of genes that elicit disuse muscle atrophy via the transcription factors p50 and Bcl-3. PLoS One (2011) 6(1):e16171. doi:10.1371/journal.pone.0016171

69. Merritt EK, Thalacker-Mercer A, Cross JM, Windham ST, Thomas SJ, Bamman MM. Increased expression of atrogenes and TWEAK family members after severe burn injury in nonburned human skeletal muscle. J Burn Care Res (2013) 34(5):e297-304. doi:10.1097/BCR.0b013e31827a2a9c

70. Lazaar AL, Miller BE, Francombe G, Duvoix A, Natanek SA, Polkey MI. No TWEAK for COPD. Eur Respir J (2013) 42(4):1142-4. doi:10.1183/09031936. 00035413

71. von Walden F, Jakobsson F, Edstrom L, Nader GA. Altered autophagy gene expression and persistent atrophy suggest impaired remodeling in chronic hemiplegic human skeletal muscle. Muscle Nerve (2012) 46(5):785-92. doi:10.1002/ mus. 23387

72. Zheng TS, Burkly LC. No end in site: TWEAK/Fn14 activation and autoimmunity associated-end-organ pathologies. J Leukoc Biol (2008) 84(2):338-47. doi:10.1189/jlb.0308165
73. Termin A, Staron RS, Pette D. Myosin heavy chain isoforms in histochemically defined fiber types of rat muscle. Histochemistry (1989) 92(6):453-7. doi:10.1007/BF00524756

74. Ennion S, Sant'ana Pereira J, Sargeant AJ, Young A, Goldspink G. Characterization of human skeletal muscle fibres according to the myosin heavy chains they express. J Muscle Res Cell Motil (1995) 16(1):35-43. doi:10.1007/BF00125308

75. Wang YX, Zhang CL, Yu RT, Cho HK, Nelson MC, Bayuga-Ocampo CR, et al. Regulation of muscle fiber type and running endurance by PPARdelta. PLoS Biol (2004) 2(10):e294. doi:10.1371/journal.pbio.0020294

76. Gan Z, Rumsey J, Hazen BC, Lai L, Leone TC, Vega RB, et al. Nuclear receptor/microRNA circuitry links muscle fiber type to energy metabolism. J Clin Invest (2013) 123(6):2564-75. doi:10.1172/JCI67652

77. Picard M, Hepple RT, Burelle Y. Mitochondrial functional specialization in glycolytic and oxidative muscle fibers: tailoring the organelle for optimal function. Am J Physiol Cell Physiol (2012) 302(4):C629-41. doi:10.1152/ajpcell.00368. 2011

78. Cao PR, Kim HJ, Lecker SH. Ubiquitin-protein ligases in muscle wasting. Int J Biochem Cell Biol (2005) 37(10):2088-97. doi:10.1016/j.biocel.2004.11.010

79. Li JB, Goldberg AL. Effects of food deprivation on protein synthesis and degradation in rat skeletal muscles. Am J Physiol (1976) 231(2):441-8.

80. Tiao G, Lieberman M, Fischer JE, Hasselgren PO. Intracellular regulation of protein degradation during sepsis is different in fast- and slow-twitch muscle. Am J Physiol (1997) 272(3 Pt 2):R849-56.

81. Sato S, Ogura Y, Mishra V, Shin J, Bhatnagar S, Hill BG, et al. TWEAK promotes exercise intolerance by decreasing skeletal muscle oxidative phosphorylation capacity. Skelet Muscle (2013) 3:18. doi:10.1186/2044-5040-3-18

82. Finck BN, Kelly DP. PGC-1 coactivators: inducible regulators of energy metabolism in health and disease. J Clin Invest (2006) 116(3):615-22. doi:10.1172/ JCI27794

83. Uldry M, Yang W, St-Pierre J, Lin J, Seale P, Spiegelman BM. Complementary action of the PGC-1 coactivators in mitochondrial biogenesis and brown fat differentiation. Cell Metab (2006) 3(5):333-41. doi:10.1016/j.cmet. 2006.04.002

84. Lin J, Wu H, Tarr PT, Zhang CY, Wu Z, Boss O, et al. Transcriptional co-activator PGC-1 alpha drives the formation of slow-twitch muscle fibres. Nature (2002) 418(6899):797-801. doi:10.1038/nature00904

85. Handschin C, Chin S, Li P, Liu F, Maratos-Flier E, Lebrasseur NK, et al. Skeletal muscle fiber-type switching, exercise intolerance, and myopathy in PGC-1alpha muscle-specific knock-out animals. J Biol Chem (2007) 282(41):30014-21. doi:10.1074/jbc.M704817200

86. Geng T, Li P, Okutsu M, Yin X, Kwek J, Zhang M, et al. PGC-1alpha plays a functional role in exercise-induced mitochondrial biogenesis and angiogenesis but not fiber-type transformation in mouse skeletal muscle. Am J Physiol Cell Physiol (2010) 298(3):C572-9. doi:10.1152/ajpcell.00481.2009

87. Handschin C, Rhee J, Lin J, Tarr PT, Spiegelman BM. An autoregulatory loop controls peroxisome proliferator-activated receptor gamma coactivator lalpha expression in muscle. Proc Natl Acad Sci U S A (2003) 100(12):7111-6. doi:10.1073/pnas.1232352100

88. Richter EA, Hargreaves M. Exercise, GLUT4, and skeletal muscle glucose uptake. Physiol Rev (2013) 93(3):993-1017. doi:10.1152/physrev.00038.2012

89. Egan B, Zierath JR. Exercise metabolism and the molecular regulation of skeletal muscle adaptation. Cell Metab (2013) 17(2):162-84. doi:10.1016/j.cmet.2012. 12.012

90. Shi J, Jiang B, Qiu Y, Guan J, Jain M, Cao X, et al. PGC1alpha plays a critical role in TWEAK-induced cardiac dysfunction. PLoS One (2013) 8(1):e54054. doi:10.1371/journal.pone.0054054

91. Dumortier O, Hinault C, Van Obberghen E. MicroRNAs and metabolism crosstalk in energy homeostasis. Cell Metab (2013) 18(3):312-24. doi:10.1016/ j.cmet.2013.06.004

92. Chen JF, Callis TE, Wang DZ. microRNAs and muscle disorders. J Cell Sci (2009) 122(Pt 1):13-20. doi:10.1242/jcs.041723

93. McCarthy JJ, Esser KA. MicroRNA-1 and microRNA-133a expression are decreased during skeletal muscle hypertrophy. J Appl Physiol (2007) 102(1):306-13. doi:10.1152/japplphysiol.00932.2006

94. Allen DL, Bandstra ER, Harrison BC, Thorng S, Stodieck LS, Kostenuik PJ, et al. Effects of spaceflight on murine skeletal muscle gene expression. J Appl Physiol (2009) 106(2):582-95. doi:10.1152/japplphysiol.90780.2008 
95. Gallagher IJ, Scheele C, Keller P, Nielsen AR, Remenyi J, Fischer CP, et al. Integration of microRNA changes in vivo identifies novel molecular features of muscle insulin resistance in type 2 diabetes. Genome Med (2010) 2(2):9. doi:10.1186/gm130

Conflict of Interest Statement: The authors declare that the research was conducted in the absence of any commercial or financial relationships that could be construed as a potential conflict of interest.

Received: 31 October 2013; accepted: 14 January 2014; published online: 27 January 2014.
Citation: Sato S, Ogura Y and Kumar A (2014) TWEAK/Fn14 signaling axis mediates skeletal muscle atrophy and metabolic dysfunction. Front. Immunol. 5:18. doi: 10.3389/fimmu.2014.00018

This article was submitted to Inflammation, a section of the journal Frontiers in Immunology.

Copyright (C) 2014 Sato, Ogura and Kumar. This is an open-access article distributed under the terms of the Creative Commons Attribution License (CC BY). The use, distribution or reproduction in other forums is permitted, provided the original author(s) or licensor are credited and that the original publication in this journal is cited, in accordance with accepted academic practice. No use, distribution or reproduction is permitted which does not comply with these terms. 